mutations to several genes, and all forms of infantile spasms may be symptomatic. The symptomatic, cryptogenic, and idiopathic classification system of epilepsy syndromes should be replaced, as recommended by the ILAE. (Berg AT et al. Epilepsia 2010;51:676-685). This review is considered a first step toward a genetic and biological classification of infantile spasms.

\title{
VIGABATRIN TRIALS AND DOSE RECOMMENDATIONS
}

Major, well-controlled trials of Vigabatrin for infantile spasms in Europe, Canada, and the Unites States are reviewed, including dose recommendations. Effective dosages ranged from 100 to $150 \mathrm{mg} / \mathrm{kg} /$ day, decreasing or eradicating spasms and eliminating the hypsarrhythmic EEG in newly diagnosed patients. Studies demonstrated long-term seizure control with no adverse effect on development and few severe adverse effects. Visual field defects were not evaluated. Time to response was within 2 weeks of initiating treatment. If the infant has not shown clinical improvement in 2 weeks, vigabatrin is discontinued and alternate treatment initiated. Treatment duration up to 6 months controlled seizures while limiting potential risks of adverse events and seizure recurrence.

A randomized, comparative trial of vigabatrin $(150 \mathrm{mg} / \mathrm{kg} /$ day $)$ and hydrocortisone $(15 \mathrm{mg} / \mathrm{kg} /$ day $)$ in 22 patients with tuberous sclerosis, found vigabatrin more efficacious and better tolerated than hydrocortisone, with $100 \%$ response during this 1- to 3-month crossover trial. Vigabatrin is recommended as first-choice treatment of infantile spasms caused by tuberous sclerosis.

In the UK Infantile Spasm Study comparing vigabatrin (100-150 mg/kg/day) with hormonal treatment (oral prednisolone $40-60 \mathrm{mg} /$ day, or intramuscular tetracosactide 0.5 $0.75 \mathrm{mg}$ [40-60 IU], a synthetic analog of ACTH, on alternate days), hormonal treatments were superior to vigabatrin in control of spasms; adverse events were common with both forms of therapy. Hormonal treatment was preferred over vigabatrin. Infants with tuberous sclerosis were excluded from this study. Follow-up studies at age 14 months and 4 years indicated equivalent spasm-freedom and developmental outcomes with vigabatrin and hormone treatment, except for infants with cryptogenic spasms; infants with no identified etiology for spasms had higher developmental scores following hormone therapy.

Except for the UK study, all investigators preferred vigabatrin as first-line therapy over hormonal. The most common adverse events with vigabatrin were sedation and irritation. Delay in time to diagnosis and treatment is associated with less favorable outcomes. (Carmant L. Vigabatrin therapy for infantile spasms: review of major trials in Europe, Canada, and the United States, and recommendations for dosing. Acta Neurol Scand Dec 2011;124 (Suppl 192):36-47). (Respond: Dr L Carmant, Division of Neurology, Universite de Montreal, CHU-Sainte-Justine, 3175 Cote Sainte-Catherine, Room 5421, Montreal, QC, Canada H3T 1C5. E-mail: lionel.carmant@umontreal.ca).

COMMENT. Vigabatrin is an irreversible GABA inhibitor, effective as adjunctive therapy in control of refractory complex partial seizures in adults and as monotherapy for infantile spasms. Clinical benefits of vigabatrin must be balanced with associated risk of peripheral visual field defects (pVFDs). (Pellock JM. Acta Neurol 
Scand 2011;124(Suppl 192):83-91). Field defects are bilateral and more pronounced nasally. Reported estimates of pVFDs are $30-50 \%$ in adults and $20 \%$ in children. Risk increases with increased dosage and duration of therapy. Vision screening is recommended at baseline, every 3 months, and at 3-6 months after discontinuation of therapy.

\section{SEIZURE-INDUCED MIOSIS}

Researchers at University of Southampton, UK report an infant with focal seizures secondary to cortical dysplasia who presented at age 4 months with bilateral pinpoint pupils as first feature of right-sided clonic seizure. EEG recording during seizures showed ictal spiking over the left central region. ECG did not show bradycardia, and there were no signs of parasympathetic activation. MRI, obtained after relapse at 13 months of age with 30 seizures daily involving the right upper limb, revealed left frontal cortical dysplasia. A cortical electrographic grid and intracranial EEG demonstrated ictal fast spike activity within the left centroparietal region, over the middle parietal gyrus. The irritative zone was located at the margin and across the area of dysplasia. The child remained seizure-free at 3 years following excision of the lesion. The origin of ictal miosis is considered secondary to activation of a cortical pupillary constrictor zone as

opposed to activation of the parasympathetic nervous system through the subcortical central autonomic network, usually resulting in mydriasis. (Sadek A-R, Kirkham F, Barker S, Gray WP, Allen D. Seizure-induced miosis. Epilepsia Dec 2011;52(12):e199e203). (Respond: Dr Ahmed-Ramadan Sadek, Division of Clinical Neurosciences, School of Medicine, University of Southampton, Tremona Road, Southampton SO16 6YD, UK. E-mail: a.sadek@soton.ac.uk).

COMMENT. Bilateral pupillary dilatation preceding, during, or after generalized convulsive or nonconvulsive seizures is common, but ictal pupillary miosis is rare. Autonomic disturbances during seizures (alterations in heart rate, blood pressure, gastrointestinal function) result from seizures within the largely subcortical and brainstem central autonomic network. The UK study demonstrates ictal miosis originating in the middle parietal gyrus juxtaposed by the superior anastomatic vein of Trolard, within the cortical central parietal region.

\section{MITOCHONDRIAL HYPERMETABOLIC NEURONS IN EPILEPSY}

Researchers at the University of Calgary and Alberta Children's Hospital, Canada studied histochemically frozen sections of 10 brain resections from 7 epileptic children (ages 2 months to 17 years). None had mitochondrial disease; 1 had tuberous sclerosis and 2 hemimegalencephaly. Preop EEG and intra-operative ECoG defined foci refractory to AEDs. Increased mitochondrial respiratory chain enzymes were demonstrated in individual neurons of hippocampal and neocortex epileptic foci. These intensely staining neurons may indicate functional hypermetabolism and represent a metabolic marker of an epileptic focus. Alpha-B-crystallin was also strongly reactive, demarcating the epileptic focus as a wide field. (Sarnat HB, Flores-Sarnat L, Hader W, Bello-Espinosa L. Mitochondrial "hypermetabolic" neurons in paediatric epileptic foci. Can J Neurol Sci 